\title{
A HIGH-POWER MEMS ELECTRIC INDUCTION MOTOR
}

\author{
Carol Livermore, Anthony Forte, Theodore Lyszezarz, Stephen D. Umans, Jeffrey H. Lang \\ Department of Electrical Engineering and Computer Science, Microsystems Technology Laboratories, and \\ Lincoln Laboratory \\ Massachusetts Institute of Technology \\ Cambridge, MA 02139
}

\begin{abstract}
An electric induction micromotor was designed and built for high-power operation. Operated at partial actuating voltage, the motor has demonstrated an air gap power in excess of $20 \mathrm{~mW}$ and torque of $3.5 \mu \mathrm{Nm}$ at speeds in excess of 55,000 rpm. The device builds on an earlier micromotor demonstrated by Frechette et al. [1]. The high power of the present motor is enabled by its lowloss, high voltage electrical stator, which also offers improved efficiency. The development of this electromechanical device is an important enabling step not only for Watt-scale micromotors, but also for the development of micro electrical generators. This paper presents the motor's design, the fabrication process that was created to meet its stringent design requirements, and its performance to date.

\section{INTRODUCTION}

Applications for Power MEMS range from generating portable electrical power, to propelling micro-scale vehicles, to driving miniature devices like pumps or fans. However, the substantial power handling potential of such devices is accompanied by substantial challenges in their development. High power also implies high levels of other characteristics, such as voltages, currents, temperatures, frequencies, or speeds.

A team at MIT is developing a family of Power MEMS devices for applications like those described above [2]. The device described here is an electric induction micromotor designed to convert electrical power into mechanical power at the Watt level. To function at these power levels, the motor is designed to operate at high voltages (about $300 \mathrm{~V}$ ), high electrical frequencies (a few $\mathrm{MHz}$ ), and high rotational speeds (greater than $1 \mathrm{Mrpm}$ ). These same design requirements, coupled with low electrical losses, would enable a micro-scale electrical generator. The purpose of the present work is to demonstrate that a micro-scale machine can be designed and fabricated to meet these specifications, and to characterize its performance over a range of operating conditions.
\end{abstract}

\section{DEVICE DESIGN}

The motor's actuating principle is electric induction, which is illustrated in Figure 1. The motor has two fundamental components, a spinning rotor disk and a fixed stator plate. Covering the stator surface is an array of radial electrodes, arranged like spokes on a wheel, which are excited with ac voltages to create a traveling wave of potential along the stator surface. Facing the stator is the spinning rotor, which is coated with a thin film of a poorly conducting material. The stator and rotor are separated by a $3 \mu \mathrm{m}$ air gap. The electric potential on the stator induces image charges in the rotor film. As the stator excitation travels along, the image charges follow, conducting through the rotor film. By design, the conductivity of the rotor film is low enough that the rotor charges lag behind the stator excitation. This generates a tangential electric field that pulls on the rotor charges to create a torque, thereby converting electrical power into mechanical power. Further description and models of electric induction machines may be found in [3] and [4]. A microscale electric induction motor has the potential to operate at higher power density than would a macro-scale electric machine because the small gap between rotor and stator can sustain higher electric fields without electrical breakdown than could a larger gap.

The motor's rotor and actuating mechanism are integrated into a micromachined silicon static structure. The complete motor comprises a stack of five silicon wafers; a schematic cross-section of the device is shown in Figure 2. Two of the wafers are patterned with thick and thin films to create the motor's actuating elements, the electrical stator and the electrical rotor. The electrical stator is a planar array of 786 radial electrodes wired into six phases by a separate layer of interconnections, forming 131 pole pairs. On the side of the rotor facing the stator, the spinning rotor disk houses a thin layer of moderately boron-doped polysilicon, which serves as the rotor film. The back side houses a set of turbine blades; these can be driven with compressed air to characterize the rotational performance of the device in the absence of electrical excitation. In related applications, blades could form the working part of a compressor or pump, or turbine blades could drive rotation of the disk for electrical power generation. The wafers are deep-etched to define the freely moving, $4.2 \mathrm{~mm}$ diameter, $550 \mu \mathrm{m}$ thick rotor, along with its system of supporting gas bearings and auxiliary fluid systems. In operation, the rotor is supported both axially and in-plane on films of externally pressurized air that are injected into narrow gaps between the rotating and stationary components. Such bearings have previously been demonstrated to support rotation of a similar microdevice at speeds well over $1 \mathrm{Mrpm}$ [5].

Achieving high-power, low-loss motor performance depends on maximizing the capacity to convert electrical power to mechanical power and maximizing efficiency. Analytical system modeling was used to examine the tradeoffs among operating point, conversion capacity, ease of fabrication, and losses in the device and electronics. The results show that minimizing stray capacitance between the electrical components and the substrate is critical both for power conversion capacity and to minimize electrical losses. To meet this requirement, the design calls for

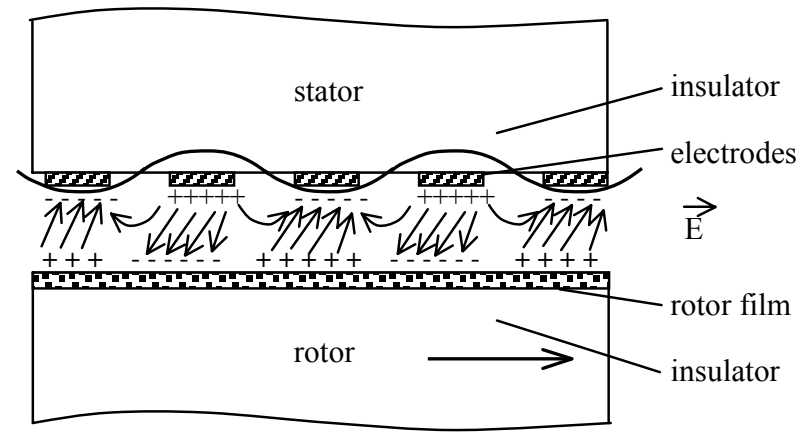

Figure 1. Schematic diagram of motor operation. A traveling wave of potential on the stator electrodes induces image charges in the rotor film; the resulting tangential electric field spins the rotor. 


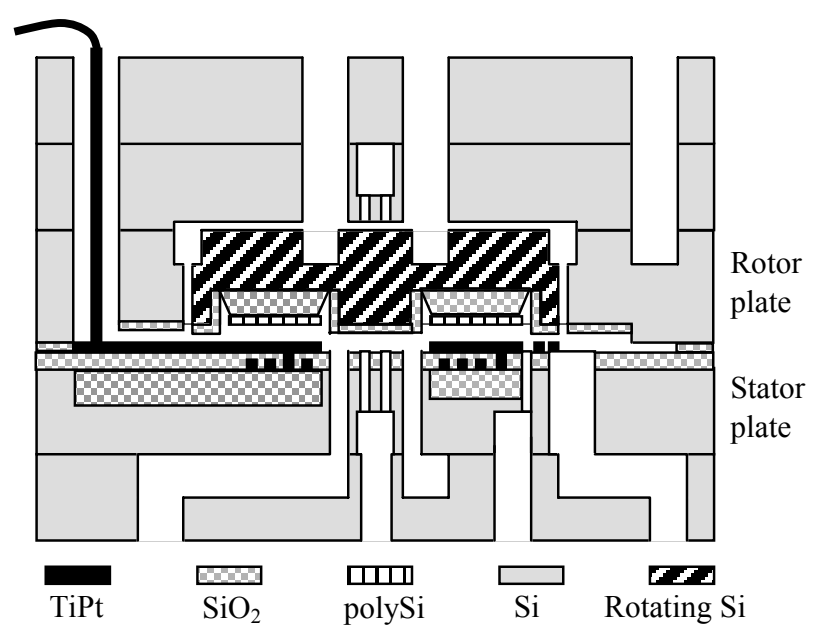

Figure 2. Cross-sectional schematic diagram of motor showing its five constituent through-etched wafers. Also shown is the integration of the electrical stator and rotor into the stack.

electrical insulating layers that are tens of micrometers thick. The thick insulators reduce parasitic capacitance and the losses associated with reactive power flowing through them. However, depositing and patterning such films complicates fabrication and makes wafer bonding more difficult. Second, modeling shows that it is imperative to be able to sustain high electric fields of about $10^{8} \mathrm{~V} / \mathrm{m}$ at high frequencies between components separated by several micrometers without suffering electrical breakdown. The higher design frequencies enable higher power, and output power increases as the square of the operating voltage. However, this demands cleanliness and smooth line edges on the electrical stator; defects contribute to early failure from electrical breakdown. In addition, the electrodes and interconnects must be fabricated of a low-resistivity metal to minimize resistive electrical losses. This requirement must be balanced against the thermal and chemical stability that the microfabrication process demands.

\section{FABRICATION}

The rotor and fluid flow paths are defined in the device's five constituent silicon wafers by double-sided, aligned deep reactive ion etching. This portion of the fabrication is similar to the process described in [5]. Figure 3 shows a photograph of dies from each of the five wafer levels; only four dies are visible because two of the levels are already wafer bonded together. The device is designed to use fusion bonding for the final assembly. However, the device described here was instead assembled at the die level by clamping the individual dies together in an aluminum/acrylic package. The o-rings that create sealed fluidic connections between the outer ports of the device and the inner surface of the package also provide compression to minimize leakage between plates. This provides an experimental advantage: the device can be disassembled for inspection, cleaning, or to perform diagnostics.

The design requirements of low losses and high electrical fields primarily impact the fabrication of the electrical rotor and the electrical stator. While the rotor fabrication process is similar to that used in [1], the stator fabrication process is significantly different. For this reason, the emphasis here will be on the stator fabrication process.

Both stator and rotor elements are fabricated on 10 to $20 \mu \mathrm{m}$ thick oxide layers to minimize stray capacitance of the electrical elements to the substrate. To minimize wafer bow while maintaining a planar wafer surface, the thick oxide layers are limited to isolated oxide islands that are embedded in the silicon substrate. A cross-section of an embedded oxide island in the stator is shown in Figure 4A. The island concept has been demonstrated previously [6], but in the present stator, the islands are fabricated by a different and direct technique. Thick oxides are deposited in etched recesses in a silicon substrate using a liftoff process, producing a nearly planar oxide surface. To achieve this, $20 \mu \mathrm{m}$ deep pits are first etched in the silicon substrate. An aluminum layer with micrometer-scale thickness is deposited and patterned to cover the upper silicon surface but not inside the pits; this serves as the sacrificial layer for the liftoff step. Next, $20 \mu \mathrm{m}$ of TEOS is deposited over the wafer surface. Liftoff is accomplished by a prolonged $\mathrm{HCl}$ etch; the field oxide breaks off neatly, leaving micrometer-scale stubs at the pit edges. If necessary, the stubs may then be minimized by CMP.

The stator electrodes and interconnections are fabricated as two interconnected $0.3 \mu \mathrm{m}$ thick platinum levels with titanium adhesion layers. Each stator electrode is $900 \mu \mathrm{m}$ long, tapers from $11 \mu \mathrm{m}$ to $4 \mu \mathrm{m}$ wide, and is separated from its neighboring electrodes by just $4 \mu \mathrm{m}$. The two platinum layers are fabricated by a liftoff process on top of the thick oxide islands in the silicon substrate, as shown in Figure 4. The upper platinum layer forms the stator's electrodes; a separate underlying layer of platinum interconnection rings groups the electrodes into six phases. The electrodes and interconnects are separated by a $1 \mu \mathrm{m}$ thick layer of TEOS oxide. Electrical contact between the two layers is established by via contacts etched in the interlayer oxide. The low resistivity of the platinum electrodes and interconnects helps to minimize electrical losses. In addition, their very smooth, liftedoff edges have been experimentally demonstrated to consistently resist electrical breakdown up to the design point of nearly $10^{8}$ $\mathrm{V} / \mathrm{m}$ at $\mathrm{MHz}$ frequencies.

\section{EXPERIMENTAL RESULTS}

The motor's performance is characterized as a function of stator excitation voltage and frequency. The hydrostatic gas bearings are first pressurized to float the rotor on a film of air; the stator is then excited with a traveling wave of electric potential to actuate the motor. A fiber optic sensor is inserted into a port in the device to monitor the passage of deep-etched features on the rotor. A spectrum analyzer processes the sensor's output to yield the motor's rotational speed. The motor operates at no load; its speed

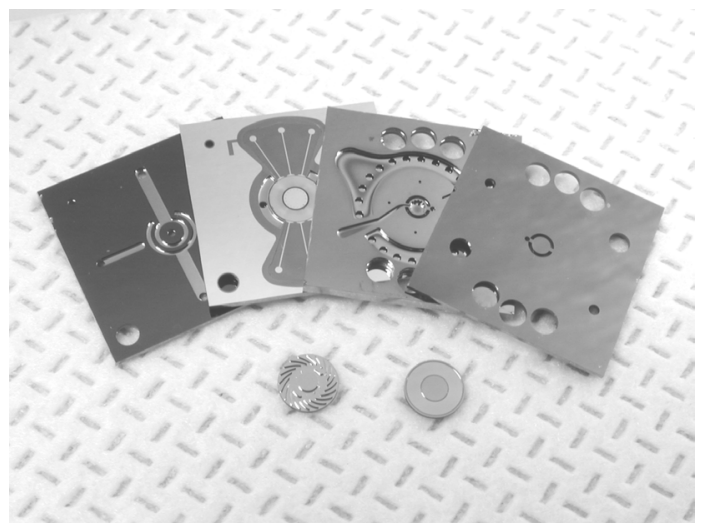

Figure 3. Fabricated die levels of the micro-motor. The electrical stator is second from the left, and both sides of the rotor are shown below. 
is set by the balance between the motor's actuating torque and the viscous drag on the spinning rotor. The viscous torque is measured as a function of rotational speed. The motor's torque and power are then extracted from the measured rotational speed using the experimentally determined load line.

The motor's load line is measured by abruptly removing the stator excitation and monitoring the rotor's deceleration with the optical speed sensor. From these data and the known rotor inertia, we can extract the decelerating viscous torque. This yields a fixed viscous torque constant of $(5.6 \pm 1) \times 10^{-4} \mu \mathrm{N} \cdot \mathrm{m} \cdot \mathrm{s}$ plus a constant $0.2 \pm 0.01 \mu \mathrm{N} \cdot \mathrm{m}$ component of the torque that reflects mechanical rubbing of a piece of debris in one of the thrust bearings.

Figure 5 plots the motor's torque and power measured as a function of electrical frequency for a fixed stator excitation voltage of $45 \mathrm{~V}_{\text {peak }}$. The solid lines are fits of a model $[3,4]$ to the data, with the rotor film conductivity and motor gap as adjustable parameters. The measured torque and power both peak at about

A)

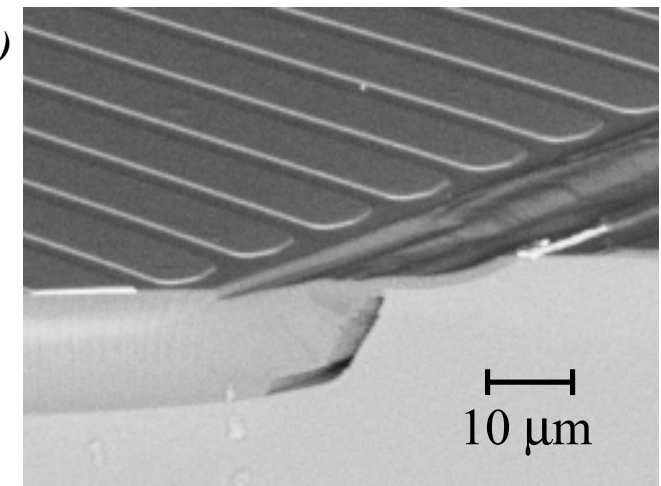

B)
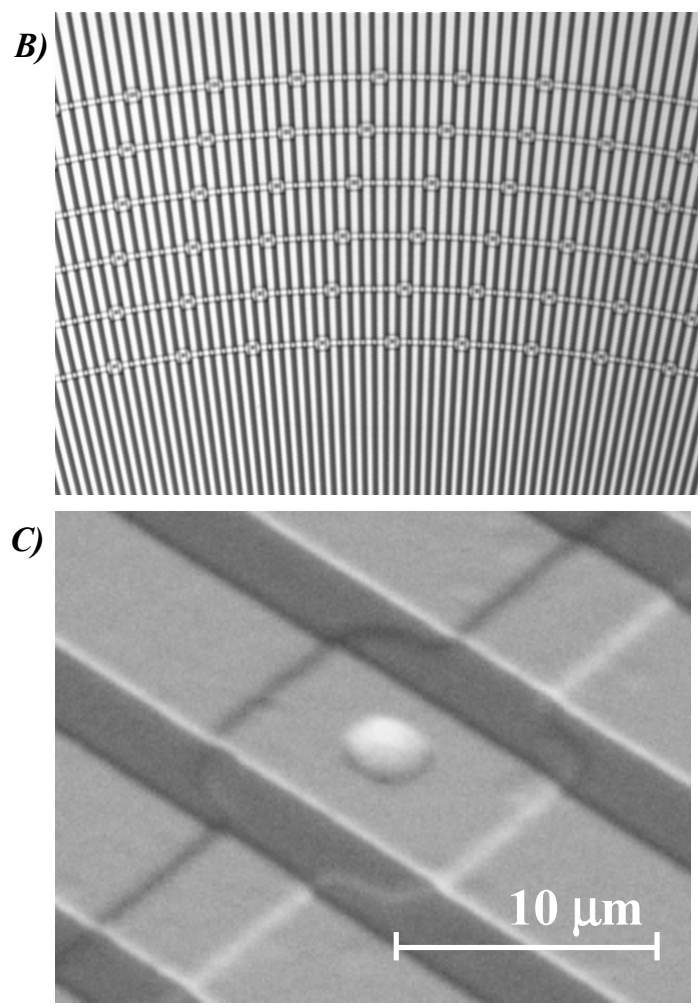

Figure 4. A) SEM micrograph of stator cross-section, showing electrodes on a recessed oxide island. B) Optical micrograph of the stator structure, showing electrodes connected by interconnection rings. C) SEM micrograph of electrodes and interconnection ring, connected by an electrical via.
A)
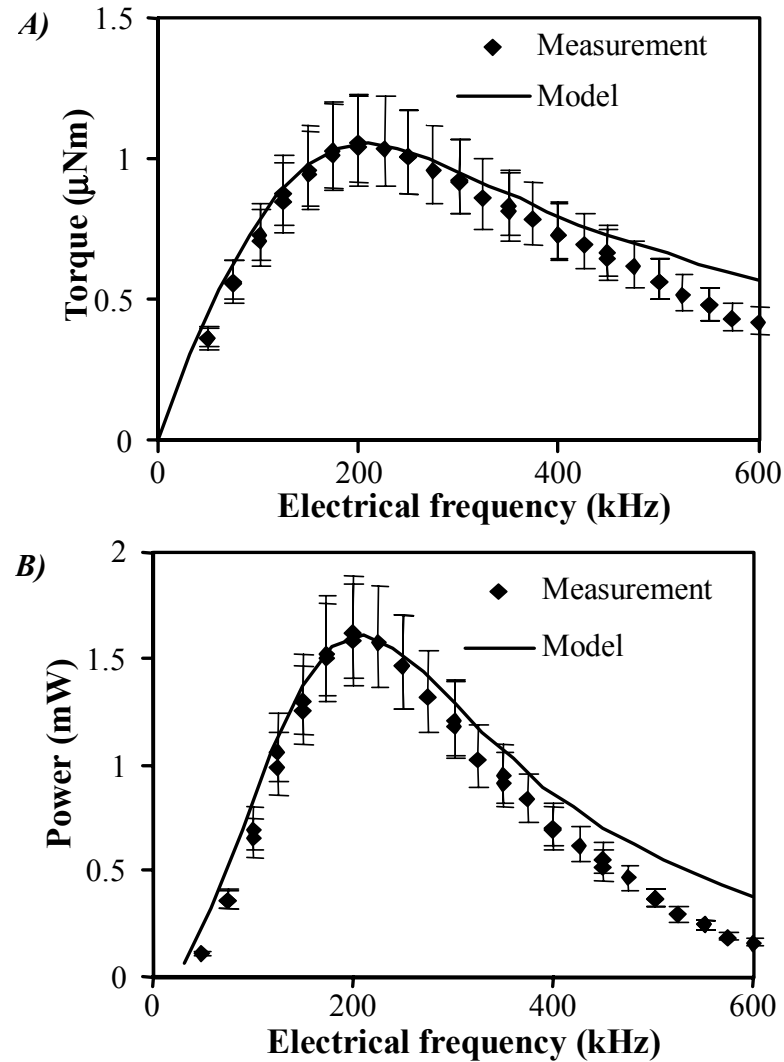

Figure 5. A) Measured motor torque and B) measured motor power as a function of electrical excitation frequency at a fixed stator excitation voltage of $45 \mathrm{~V}$.

$200 \mathrm{kHz}$. The peak frequency reflects the resistivity of the moderately doped polysilicon film on the rotor surface, and corresponds to a rotor film sheet conductance of $1.2 \mathrm{nS} / \mathrm{square}$. This is lower than the target conductance of $3 \mathrm{nS} /$ square for this device and likely reflects the omission of high temperature wafer bonding anneals that would promote grain growth in the polysilicon film. The film resistivity impacts the device's maximum possible power output; optimal power is obtained when the rotor film resistivity is well matched to a MHz-range operating frequency.

The peak amplitudes of the measured torque and power reflect the gap between the rotor and stator; more power is converted across a smaller air gap. The data correspond to a motor gap of $1.85 \mu \mathrm{m}$. This is smaller than the design value of $3 \mu \mathrm{m}$, enabling more power, and it is consistent with the as-fabricated device geometry and the measured viscous torque. Using these values for motor gap and rotor film resistivity, measured and predicted values of torque and power match well.

Figure 6 plots the motor's torque and power measured as a function of the amplitude of the actuating voltage at a fixed electrical frequency of $200 \mathrm{kHz}$. The torque starts to increase quadratically with voltage, as expected; the trend has an inflection point because the rotor speed cannot exceed the relatively low synchronous speed of the stator excitation at $200 \mathrm{kHz}$. The maximum measured torque is $3.5 \mu \mathrm{Nm}$ at a speed of over 55,000 rpm for an excitation voltage of $95 \mathrm{~V}_{\text {peak. }}$. The maximum air gap power attained exceeds $20 \mathrm{~mW}$, corresponding to a power density of $16 \mathrm{~kW} / \mathrm{m}^{3}$. The solid line is the device performance calculated from the model using the motor gap and rotor film resistivity 

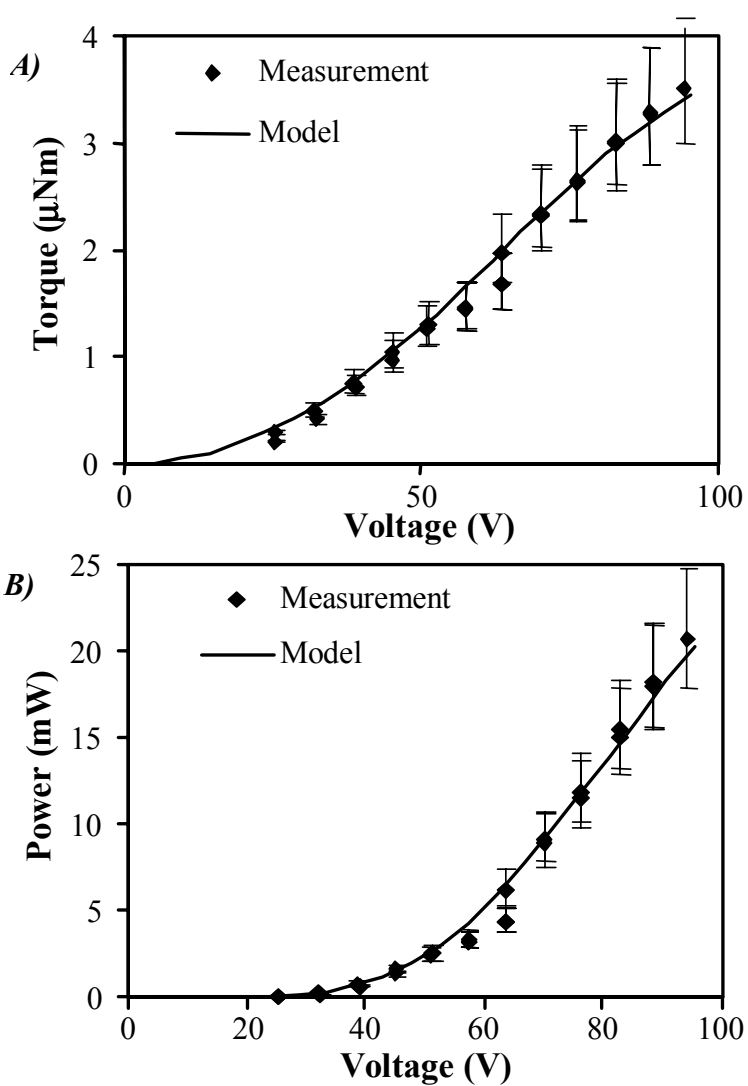

Figure 6. A) Measured motor torque and B) measured motor power as a function of stator excitation voltage at a fixed stator excitation frequency of $200 \mathrm{kHz}$.

determined above. Using the same parameters, the data again agree well with theory.

The maximum actuating voltage in these tests was not limited by electrical breakdown, but rather by the high rotational speeds that are necessary to balance a large actuating torque against viscous drag under no-load operating conditions. In the experiments to date, speed has been limited to ensure an opportunity to characterize devices fully at lower power levels before risking bearing failure or structural damage at higher speeds, which have affected other devices. However, the stators' demonstrated ability to withstand design fields suggests that the motors will be able to attain design voltage and power levels as well.

This micromotor compares well with macro-scale magnetic integral horsepower industrial motors on performance metrics like the shear stress on the rotor and the ratio of this shear stress to the normal pressure. The maximum shear stress attained so far by our electric motors is about $210 \mathrm{~N} / \mathrm{m}^{2}$, at approximately half of the design electric field. If these results are scaled up to full field, as tests on the electrical stators indicate should be possible, the shear stress would be about $800 \mathrm{~N} / \mathrm{m}^{2}$, as compared with about 7,000 $\mathrm{N} / \mathrm{m}^{2}$ for a typical integral horsepower magnetic induction machine [7]. The ratio of shear stress to normal pressure attained in our electric motors so far is about $2 \%$, comparable to the typical magnetic induction machine described above. The micromotor's ability to harness the available energy density in its air gap is comparable to that of a macro-scale magnetic machine.

\section{CONCLUSIONS}

An electric induction micromotor designed for high-power operation was fabricated. Operated at partial actuating voltage, the motor demonstrated an air gap power in excess of $20 \mathrm{~mW}$ and torque of $3.5 \mu \mathrm{Nm}$ at speeds in excess of $55,000 \mathrm{rpm}$. This was enabled by the development of low-loss, high-voltage electrical stators, which have been demonstrated to successfully sustain design voltages in experiments. The electrical isolation of stator components from the rest of the device was accomplished by the incorporation of thick islands of oxide that were embedded in the silicon substrate. These islands were formed by a liftoff technique that enabled automatic and nearly complete planarization of the island oxide with the surrounding silicon surface.

\section{ACKNOWLEDGMENTS}

We gratefully acknowledge the contributions of A. Ayon, A. Epstein, L. Fréchette, A. Hoelke, P. Maki, S. Nagle, S. Senturia, C. J. Teo, and P. Warren. The devices were fabricated at MIT Lincoln Laboratory and in MIT's Microsystems Technology Laboratories. This work was supported by DARPA TTO, the Army Research Office, and the Army Research Laboratory, managed by Dr. R. Rosenfeld, Dr. T. Doligalski, and Mr. J. Hopkins respectively. The Lincoln Laboratory portion of this work was sponsored by the Defense Advanced Research Project Agency under Air Force Contract F19628-00-C-0002. Opinions, interpretations, conclusions, and recommendations are those of the authors and not necessarily endorsed by the Department of Defense.

\section{REFERENCES}

1. L.G. Frechette, S.F. Nagle, R. Ghodssi, S. D. Umans, M.A. Schmidt, J. Lang, "An Electrostatic Induction Micromotor Supported on Gas-Lubricated Bearings," Technical Digest of the $14^{\text {th }}$ IEEE International Conference on Micro Electro Mechanical Systems, Interlaken, Switzerland, 1/21-25/01, IEEE (2001), pp. 290-3.

2. A.H. Epstein et al., "Micro-Heat Engines, Gas Turbines, and Rocket Engines - The MIT Microengine Project," AIAA Paper 971773, $28^{\text {th }}$ AIAA Fluid Dynamics Conference, Snowmass Village, CO, June 29-July 2, 1997.

3. S.F. Bart and J.H. Lang, "An analysis of electroquasistatic induction micromotors," Sensors and Actuators, 20, 97 (1989).

4. S.F. Nagle, Analysis, Design, and Fabrication of an Electric Induction Micromotor for a Micro Gas-Turbine Generator, Ph.D. Thesis, Massachusetts Institute of Technology, October 2000.

5. L.G. Frechette, S.A. Jacobson, F.F. Ehrich, R. Ghodssi, R. Khanna, C.W. Wong, X. Zhang, K.S. Breuer, M.A. Schmidt, and A.H. Epstein, "Demonstration of a Microfabricated High-Speed Turbine Supported on Gas Bearings", Technical Digest of the 2000 Solid-State Sensor and Actuator Workshop, Hilton Head Isl., SC, 6/4-8/00, Transducer Research Foundation, Cleveland (2000), pp. 43-47.

6. R. Ghodssi, L.G. Frechette, S.F. Nagle, X. Zhang, A.A. Ayon, S.D. Senturia, and M.A. Schmidt, "Thick Buried Oxide in Silicon (TBOS): An Integrated Fabrication Technology for Multi-Stack Wafer-Bonded MEMS Processes," Proceedings of the $10^{\text {th }}$ International Conference on Solid-State Sensors, Sendai, Japan, June 1999.

7. T.J.E. Miller, Switched-Reluctance Motors and Their Control, Oxford University Press (1993). 